\title{
Nonlocal effects in torsional deformation
}

\author{
F. Székely, I. Groma *, J. Lendvai \\ Department of General Physics, Eötvös University, Pázmány Sétány 1/A, POB 32, H-1518 Budapest, Hungary
}

Received 22 February 1999; received in revised form 28 July 1999

\begin{abstract}
The stress distribution in torsionally deformed cylindrical polycrystalline copper samples was investigated. The strain in torsional deformation changes with distance from the torsional axis and thus the local extent of work hardening and consequently the local flow stress changes as well. The variation of the flow stress with radial position in the samples was measured by microhardness tests. It was found that except for the near-axis region the local flow stress can be correctly calculated by the Nadai evaluation from the experimentally measured torque versus torsional angle function. Hardening is observed in the axis of the torsionally deformed samples in spite of the fact that the shear strain is zero here. The hardening obtained in the axis depends approximately linearly on the torsional angle, and it increases with grain size. This effect is interpreted by introducing nonlocal effects in the continuum description. (C) 2000 Elsevier Science S.A. All rights reserved.
\end{abstract}

Keywords: Torsional deformation; Nonlocal effects; Constitutive equations

\section{Introduction}

Torsion tests are often used for the investigation of plasticity at high strains, since in this deformation mode much higher strains can be achieved than in tension or compression tests [1-4]. In case of torsion, however, the deformation is inhomogeneous inside the sample, which makes the derivation of stress-strain curves from the measured torque-angle relations difficult. A commonly accepted method for the determination of the stress-strain relation for rod-shaped cylindrical samples from torsion tests is the Nadai evaluation [5] which is based on two assumptions:

(1) The shear strain, $\gamma$ is proportional to the distance from the axis of the sample, $r: \gamma=\vartheta r$ where $\vartheta$ is the angle of torsion per unit length. (This assumption was investigated and proved experimentally by Grewe and Kappler [6].)

(2) The shear stress $(\tau)$ is determined only by the shear strain: $\tau=\tau(\gamma)$.

\footnotetext{
* Corresponding author. Tel.: +36-1-372-2802; fax: + 36-1-3722811.

E-mail address: groma@metal.elte.hu (I. Groma)
}

From these assumptions for the surface of the sample the

$\tau(\gamma=R \vartheta)=\tau_{\mathrm{N}}(\gamma)=\frac{1}{2 \pi R^{3}}\left(\vartheta \frac{\mathrm{d} M}{\mathrm{~d} \vartheta}+3 M\right)$

relation can be derived where

$M=2 \pi \int_{0}^{R} \tau(r) r^{2} \mathrm{~d} r$

is the applied torque.

At a given angle of torsion $(\vartheta=$ const. $)$ the stressstrain relation $\tau_{\mathrm{N}}(\gamma)$ determined by the Nadai evaluation from the measure $M(\vartheta)$ curve can be converted to

$\tau_{\mathrm{N}}(r)=\tau_{\mathrm{N}}(\gamma=\vartheta r)$

which describes the variation of stress as a function of $r$.

The aim of this work is to check the validity of the stress distribution determined by this method in torsionally deformed samples.

\section{Experimental procedure}

Cylindrical samples of electrolytic copper (purity $99.99 \%$ ), with a diameter of $4 \mathrm{~mm}$, and a gauge length 
of $40 \mathrm{~mm}$ were used for torsion and tension tests. The samples for the stress distribution measurements were annealed at $600^{\circ} \mathrm{C}$ for $1 \mathrm{~h}$ to get a fully recrystallized and untextured structure with an average grain size of $20 \mu \mathrm{m}$. Additional measurements were made on samples with grain sizes of 50 and $75 \mu \mathrm{m}$, which were obtained by annealing at 800 and $900^{\circ} \mathrm{C}$, respectively. The tension tests were carried out in an INSTRON machine at a constant crosshead velocity of $2 \mathrm{~mm}$ $\min ^{-1}$.

For torsion tests, a free-end torsion machine was used, in which the sample is deformed at a constant angular rate. This gives a constant strain rate on the surface of the sample, which was $0.014 \mathrm{~s}^{-1}$ in the measurements presented in this paper. During the test the actual torque, the torsional angle and the length change of the samples were measured.

Microhardness measurements were carried out on the cross-section of the deformed samples. After torsion or tension the samples were cut at right angles to the axis of the sample. The surface was mechanically polished and etched for the microhardness tests. The microhard-

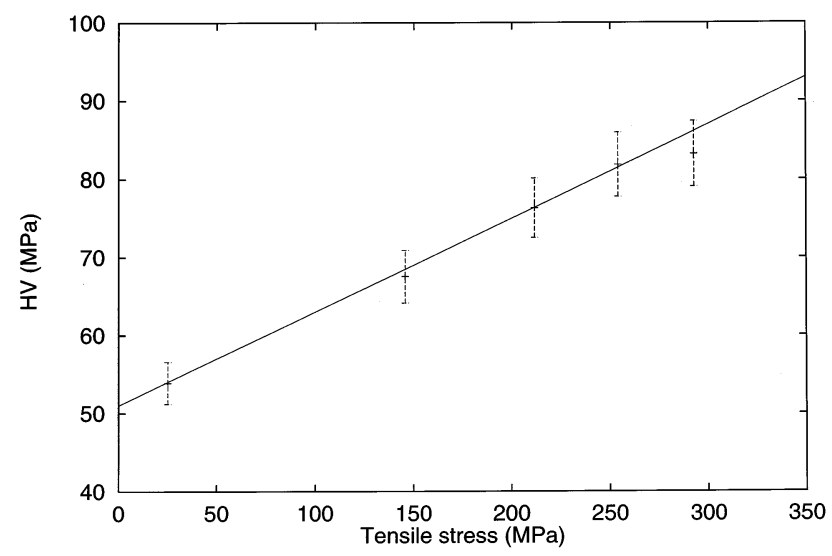

Fig. 1. The relation between the microhardness and the flow stress in the case of tension.

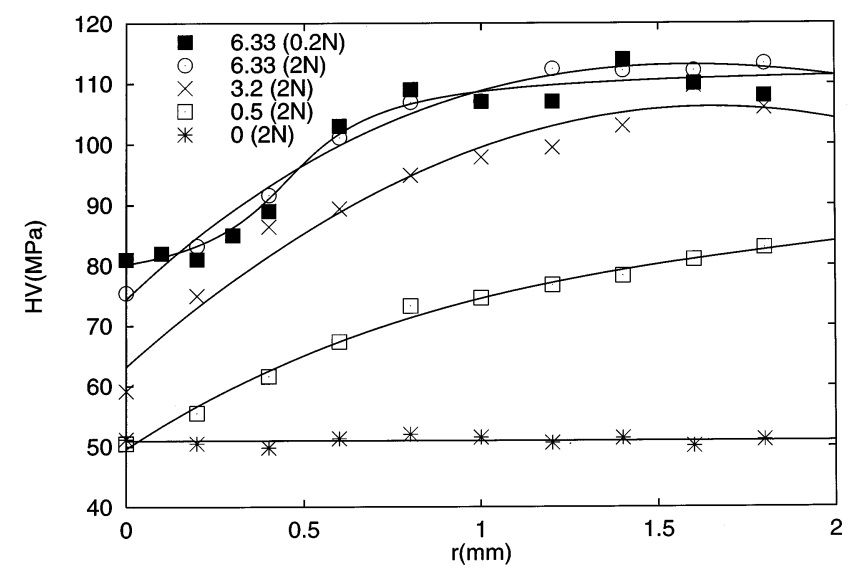

Fig. 2. The microhardness vs $r$ in samples deformed in torsion up to the indicated $\gamma=\vartheta R$ surface shear strains. ness measurements were performed on a Leitz hardness tester, with a constant load of $2 \mathrm{~N}$, resulting indentation sizes of $50-100 \mu \mathrm{m}$, and a Shimadzu DUH-202 dynamic hardness tester with a maximum load of 0.2 $\mathrm{N}$, causing indentation sizes of $10-20 \mu \mathrm{m}$.

\section{Results}

In torsional deformation the strain changes as a function of position along the radius of cylindrical samples, consequently the work hardening varies along the radius. According to the Nadai-method the local stress distribution $\tau_{\mathrm{N}}(r)$ can be determined from Eqs. (1)-(3). Using the von-Mises relation [2] this can be converted into equivalent tensile stress:

$\sigma_{\mathrm{N}}(r)=\sqrt{3} \tau_{\mathrm{N}}(r)$

The microhardness, which is an affine function of the yield stress, was measured on the cross-section of the samples as a function of $r$ to determine the distribution of the local flow stress developing during torsion. To scale the relation between the microhardness, HV, and the equivalent tensile stress, $\sigma$, microhardness measurements were carried out on the cross-section of tensile deformed samples. The $\sigma$ versus HV relation obtained (Fig. 1) can be described with the linear relation:

$\mathrm{HV}=51+0.12 \sigma$

The microhardness versus radius (distance from the axis) curve of an undeformed sample as well as of three samples deformed up to different amounts of $\gamma=\vartheta R$ surface strain are shown in Fig. 2. The microhardness of the deformed samples is monotonously increasing from the axis $(r=0)$ towards the surface $(r=2000 \mu \mathrm{m})$. It is important to note that the microhardness of the torsionally deformed samples at $r=0$ (where the shear strain according to the $\gamma=\vartheta r$ relation should be zero) increases relative to the hardness of the undeformed sample with increasing surface strain. This is in contradiction with the assumptions of traditional local plasticity, like assumption No. (2) of the Nadi evaluation [5] according to which the local flow stress depends only on the local strain.

The maximum length of the diagonal of the Vickers microhardness indentation in the present experiments was smaller than $100 \mu \mathrm{m}$, consequently taking into account the plastically deformed zone around the indentation the microhardness value is a measure of the average local flow stress in a region of 100-200 $\mu \mathrm{m}$ diameter. By applying Eq. (3) it is found that the shear strain in the axis is zero, at $r=100 \mu \mathrm{m}$ it is about 0.3 (in the case of $\gamma=6.33$ surface strain). Averaging the local flow stress for a region of $100 \mu \mathrm{m}$ around the axis on the basis of Fig. 1 and from the stress-strain curve of Fig. 3 the microhardness at the $r=0$ position should 


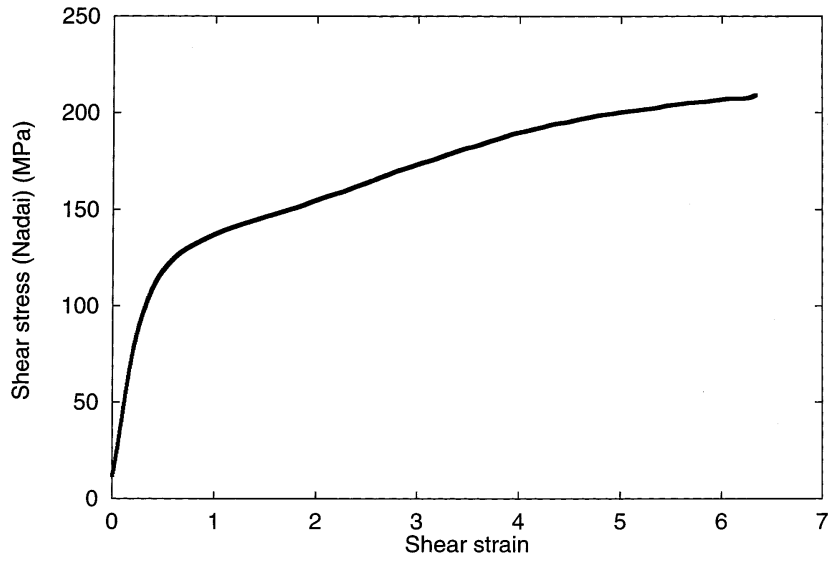

Fig. 3. Shear stress-shear strain curve determined by the Nadaimethod from the experimentally measured $M(\vartheta)$ function.

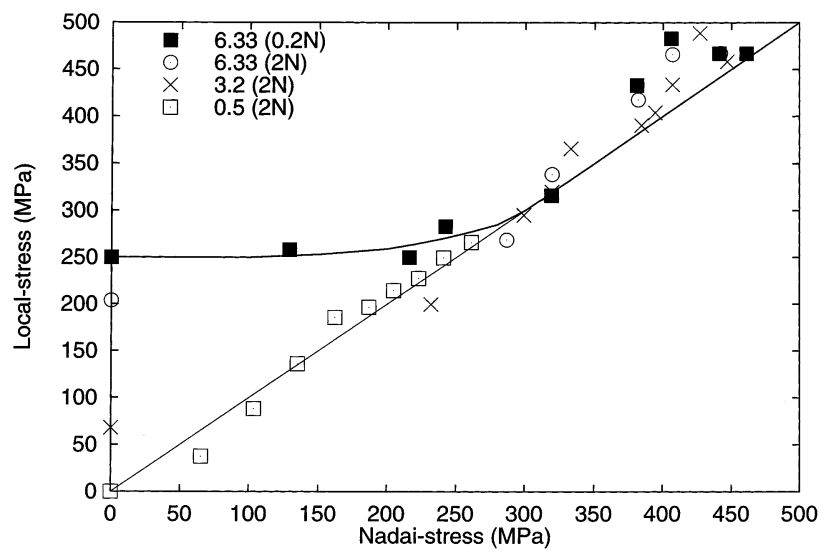

Fig. 4. The relation between the Nadai-stress and the measured local stress distribution in the torsionally deformed samples.

be about $57 \mathrm{MPa}$, i.e. an increase of only $6 \mathrm{MPa}$ relative to the undeformed value instead of the measured $25 \mathrm{MPa}$. This means that the Nadai-method can not account for the hardening observed at the $r=0$ position in Fig. 2.

The stress distribution in the case of $\gamma=6.33$ was also measured by a smaller load of $0.2 \mathrm{~N}$ resulting indentation sizes of $10-20 \mu \mathrm{m}$. In this case the hardened zones around indentations are much smaller allowing the measurements of more points near the axis, without significant interference between the independent indentations. The result of these tests are also shown in Fig. 2.

Using Eq. (5) the local microhardness values of Fig. 2. can be converted into local (equivalent) stress values: $\sigma_{\text {loc }}$. If the assumptions of the Nadai-method were valid these values should be equal to $\sigma_{\mathrm{N}}(r)$. For the calculation of the Nadai-stresses one has to take into account that at higher torsional strains due to the microstructural changes (texture development, dislocation cells, subgrains) the von-Mises relation [2] between the shear stress and the equivalent tensile stress is no longer valid.
A recent model for large strain work hardening of Estrin et al. [7] that taking into account both dislocation strucures and texture changes, suggests that during torsion the Taylor factor changes from 1.65 (vonMises) to 1.52 at the and of stage IV. Taking into account this change the $\sigma_{\mathrm{N}}$ versus $\sigma_{\text {loc }}$ diagram can be plotted (Fig. 4). By comparing the $\sigma_{\mathrm{loc}}$ and $\sigma_{\mathrm{N}}$ stresses in Fig. 4 it can be seen that apart from the points in the axis region the measured local stresses are close to the Nadai-stress proving that in those regions the two assumptions of the Nadai-method are valid. The size of the region where the measured local stresses are significantly larger than the Nadai-stresses is approximately $300 \mu \mathrm{m}$ (Fig. 2), while the grain size is $20 \mu \mathrm{m}$. It indicates that the excess hardening is not a simple single grain effect.

\section{Discussion}

The deviation from the Nadai-stress is the consequence of the inhomogeneous deformation mode, where the microstructure of the sample becomes important. To interpret the results in a continuum plasticity theory we adopted the mathematical formalism of the recently developed strain gradient plasticity theories [8-12]. The purpose of these theories is to account for the effect of microstructure in inhomogeneous plastic deformation using continuum plasticity theories. In these theories an internal length scale parameter is introduced in continuum plasticity and it is assumed that the stress in a deformed material depends not only on the local strain, but also on the gradients of strain. There are two different approaches developed so far for taking into account the influence of strain gradients. Aifantis proposed the first gradient theory [8-11] in which, independently from any microscopical mechanism, the invariants of the strain gradients are added to the conventional (only strain dependent) stress. The general form of the stress-strain relation up to second order gradient terms is the following:

$\tau=f(\gamma)-c \nabla^{2} \gamma-\bar{c}(\nabla \gamma)(\nabla \gamma)$

where the $c$ and $\bar{c}$ constants are proportional to the square of some internal length scale (e.g. grain size) and $f(\gamma)$ is the 'conventional stress'.

The other gradient theory proposed by Fleck et al. [12] is based on the concept of geometrically necessary dislocations [13]. In this approach to take into account gradient terms an effective strain measure $\bar{\epsilon}$ depending on the first order invariants of the strain $\bar{\epsilon}_{\mathrm{e}}$ and the strain gradient, $\chi_{\mathrm{e}}$, and an effective stress measure, $\Sigma$ are introduced in the following way:

$\bar{\epsilon}=\sqrt{\epsilon_{\mathrm{e}}^{2}+l^{2} \chi_{\mathrm{e}}^{2}}$

and 
$\Sigma=\frac{\mathrm{d} w}{\mathrm{~d} \bar{\epsilon}}$

where $l$ is the internal material length scale an $w$ is the strain energy density.

The internal length scale parameter which occurs in both strain gradient theories should be some microstructural length, such as grain size in the case of polycrystals, or interparticle spacing in the case of a composite structure.

Applying the formalism of Fleck et al. [12] for the case of the torsional measurements the effective strain measure, $\bar{\epsilon}$ has the form [12]:

$\bar{\epsilon}=\vartheta \sqrt{\frac{r^{2}}{3}+l^{2}}$

where $l$ is now the grain size. It follows that $\bar{\epsilon}(r=0) \neq 0$ consequently the stress measure does not disappear in the axis, which is in agreement with the experimental results.

According to Eq. (8) the work done by the external torque increases the strain energy as [12]:

$\int_{0}^{\vartheta} M(\vartheta) \mathrm{d} \vartheta=\iint_{V, 0}^{\bar{\epsilon}} \Sigma(\bar{\epsilon}) \mathrm{d} \bar{\epsilon} \mathrm{d} V$

Differentiating this expression with respect to $\vartheta$ :

$M=2 \pi \int_{0}^{R} r \sqrt{\frac{r^{2}}{3}+l^{2}} \Sigma(r) \mathrm{d} r$

To see the connection between $\Sigma(\bar{\epsilon})$ and the Nadaistress let one define an effective shear stress $\left(\tau_{\text {eff }}\right)$ giving the same torque on the surface as $\Sigma(\bar{\epsilon})$ by the following expression:

$M=2 \pi \int_{0}^{R} \tau_{\mathrm{eff}}(r) r^{2} \mathrm{~d} r$

From (11) and (12) it is obtained that:

$\tau_{\text {eff }}(r)=\frac{1}{r} \Sigma(r) \sqrt{\frac{r^{2}}{3}+l^{2}}$

Far from the torsional axis $r^{2} \gg l^{2}$ the effective shear stress is

$\tau_{\text {eff }}=\frac{\Sigma}{\sqrt{3}}$

giving back the von-Mises relation between $\tau_{\text {eff }}$ and $\Sigma$.

Eq. (12) from which the effective shear stress $\tau_{\text {eff }}$ was calculated is of the same form as Eq. (2), from which the $\tau_{\mathrm{N}}$ Nadai-stress is obtained. In the case of $\tau_{\mathrm{N}}$, however, only local strengthening is considered, consequently if there are no gradient effects or if they are negligible, $\tau_{\mathrm{N}}$ is approaching $\tau_{\text {eff }}$. In the case of $r^{2} \gg l^{2}$ the gradient effects are negligible (according to [12] $\Sigma$ and $\bar{\epsilon}$ should give the 'conventional' stress and strain) so in this case: $\tau_{\text {eff }}=\tau_{\mathrm{N}}=\frac{\Sigma}{\sqrt{3}}$

Thus in this region the $\Sigma$ stress measure (which is experienced also by the microhardness) and the $\sigma_{\mathrm{N}}(r)=\sqrt{3} \tau_{\mathrm{N}}(r)$ equivalent Nadai-stress are equal, as is also confirmed by the results shown in Fig. 4. Consequently in the case of sample diameters considerably larger than $l$ the nonlocal effects hardly influence the $M(\vartheta)$ function.

In contrast to this, near the axis where $r^{2} \ll l^{2}$ gradient effects are not negligible, $\tau_{\text {eff }}$ (and $\Sigma$ ) may differ significantly from $\tau_{\mathrm{N}}$ (see the points at $\sigma_{\mathrm{N}}=0$ Fig. 4).

In this case using a series expansion for the squareroot in Eq. (13):

$\tau_{\text {eff }}=\frac{1}{r} \Sigma(r)\left(1+\frac{r^{2}}{6 l^{2}}\right)$

is obtained.

The explicit form of the $\Sigma(\bar{\epsilon})$ constitutive relation is not known, nevertheless its initial behaviour can be investigated. If the derivative of the $\Sigma(\bar{\epsilon})$ function is finite at $\bar{\epsilon}=0$ than for small values $\bar{\epsilon}$ :

$\Sigma(\bar{\epsilon})=\Sigma_{0} \bar{\epsilon}$

where $\Sigma_{0}$ is the finite initial slope.

If the initial derivative is infinite:

$\Sigma(\bar{\epsilon})=\Sigma_{0} \bar{\epsilon}^{N}$

where $N<1$.

From the linear Eq. (17), using Eq. (9):

$\Sigma(r)=\Sigma_{0} \vartheta l$

and so with Eq. (13):

$\tau_{\text {eff }}=\Sigma_{0} \frac{\vartheta l^{2}}{r}$

For the power law relation given by Eq. (18):

$\Sigma(r)=\Sigma_{0} \vartheta^{N} l^{N}$

and

$\tau_{\text {eff }}=\Sigma_{0} \frac{\vartheta^{N} l^{N+1}}{r}$

Since $\Sigma$ is the stress measured by the microhardness, the hardening in the axis, $\Delta \mathrm{HV}=\mathrm{HV}(r=0)-\mathrm{HV}_{0}$ (where $\mathrm{HV}_{0}$ is the hardness of the undeformed sample) should follow either Eq. (19) or Eq. (21).

As visible in Fig. 2, $\Delta \mathrm{HV}$ increases with the torsional angle, $\vartheta$, i.e. with increasing $\gamma=\vartheta r$ surface shear strain. The dependence of $\Delta H V$ on $\vartheta$ is approximately linear and definitely not below linear. Consequently the behaviour described by Eq. (18) can be ruled out. The experimentally measured Nadai-stress versus shear strain curve (Fig. 3) also suggests a finite initial slope.

Additional measurements were made on samples with average grain sizes of 50 and $75 \mu \mathrm{m}$ to illustrate the 
dependence of the hardening in the axis on the microstructural length parameter for a torsional strain $\gamma=3$. It was found that the axial hardening $\Delta \mathrm{HV}$ increases linearly within experimental error with increasing grain size (Fig. 5).

The results show that the dependence of $\Delta \mathrm{HV}$ on both $\vartheta$ and $l$ is consistent within experimental error with the linear relation given by Eq. (19) while the power law of Eq. (21) with $N<1$ contradicts the experimental results.

According to the theory of Aifantis et al. [8-11] the expression of the stress of Eq. (6) for the case of torsion gives the following:

$\tau=f(\gamma)-\frac{c \vartheta}{r}-\bar{c} \vartheta^{2}$

Using this with Eq. (2), the torque at the surface can be calculated:

$M=2 \pi \int_{0}^{R} f(\gamma) r^{2} \mathrm{~d} r-\pi c \vartheta R^{2}-\frac{2}{3} \bar{c} \pi R^{3}$

In this expression the first term is the contribution of the 'conventional' stress, while the second and third term is the effect of strain gradient on the torque.

From [8] the constant $c$ for copper of $25 \mu \mathrm{m}$ grain size is approximately $15 \mathrm{~N}$ (and $c$ is negative), while $\bar{c}$ is about $0.005 \mathrm{~N}$, so the contribution of the gradient effect at the surface is negligible compared to the 'conventional' stress.

In contrast with this, near the axis the 'conventional' stress should disappear, the third term in Eq. (23) is constant and the dominant term is $c \vartheta / r$, where $c \sim l^{2}$ [8-11]. As can be seen, this expression has the same form as that obtained for $\tau_{\text {eff }}$ in Eq. (20) by applying the theory of Fleck et al. [12] for torsion with the linear constitutive relation for $\Sigma$.

So both formalisms (Fleck et al. [12] and Aifantis et al. [8-11]) predict that in our torsion tests far from the

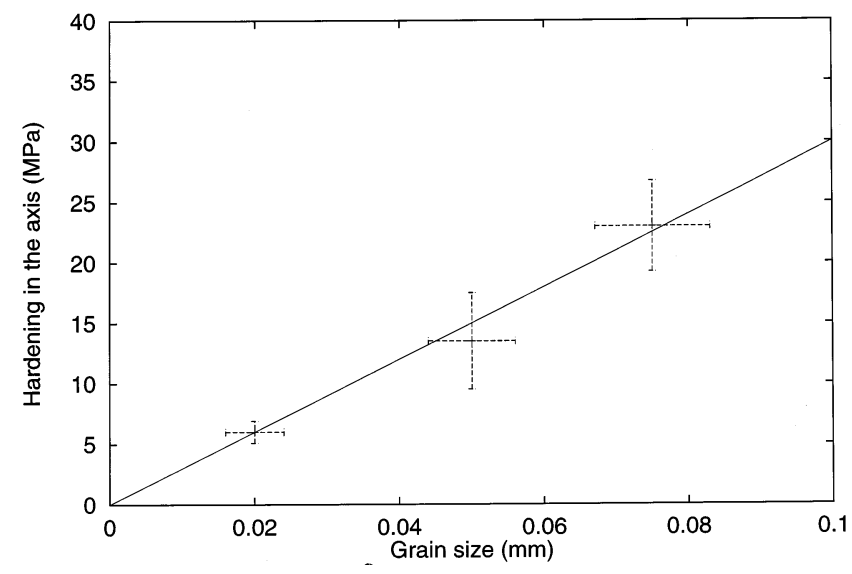

Fig. 5. The hardening in the axis vs grain size in the region of $0.02-0.1 \mathrm{~mm}$ at a fixed torsional strain of $\gamma=3$. axis (compared with the microstructural length scale $l$ ), conventional plasticity theory is valid, and the Nadaistress correctly describes the stress distribution in the samples. Near the axis, however, the experiments reveal significant deviations from conventional plasticity (i.e. the Nadai-stress). The observed discrepancy can be interpreted on the basis of both currently available strain gradient theories.

\section{Summary and conclusions}

The stress distribution developing during torsional deformation was studied by performing microhardness measurements on the cross section of the deformed samples. Work hardening was observed in the axis $(r=0)$ of the torsionally deformed cylindrical samples, which contradicts conventional local plasticity according to which the plastic deformation and consequently the work hardening at $r=0$ should be zero. The axial strengthening increases approximately linearly with the torsional angle, i. e. with the surface torsional strain.

Investigating samples of 20,50 and $75 \mu \mathrm{m}$ average grain sizes the work hardening at $r=0$ was observed to increase with grain size, the dependence being linear within the experimental accuracy and within the range of grain sizes investigated. These experimental observations, cannot be explained in the framework of the conventional constitutive description where stress is a unique function of strain. It was pointed out that to describe the experimental results nonlocal effects should be introduced in the constitutive equation. To do this the strain gradient formalism of both Aifantis [8-11] and Fleck et al. [12] can be applied. The experimental results support an initially linear $\Sigma(\bar{\epsilon})$ constitutive relation. Taking into account this linear constitutive relation for torsional deformation, in the vicinity of the axis the gradient formalism of Fleck et al. [12] gives the same result as the theory of Aifantis et al. [8-11].

Since the region where nonlocal effects are significant is of the order of $100 \mu \mathrm{m}$, these effects do not alter the stress distribution far from the axis, where the Nadaimethod gives correct results for the flow stress. In the vicinity of the axis, however, or in samples the diameter of which is comparable to the grain size [12] nonlocal effects should be taken into account, which can be achieved by applying strain gradient formalisms.

\section{Acknowledgements}

Financial support of the Hungarian Scientific Research Fund (OTKA) under contract Nos. T-030791 and T-029701 is acknowledged. 


\section{References}

[1] I. Kovács, Acta Metall. 15 (1967) 1731.

[2] J. Gil Sevillano, P. van Houtte, E. Aernoudt, Progr. Mat. Sci. 25 (1980) 69.

[3] T. Ungár, L.S. Tóth, J. Illy, I. Kovács, Acta Metall. 34 (1986) 1257.

[4] M. Zehetbauer, V. Seumer, Acta Metall. Mater. 41 (1993) 577.

[5] A. Nadai, Theory of Flow and Fracture of Solids, Vol. 2. McGraw Hill, New York, 1963, ch. 21.

[6] H.G. Grewe, E. Kappler, Phys. Stat. Sol. 6 (1964) 339.
[7] Y. Estrin, L.S. Tóth, A. Molinari, Y. Brechet, Acta Mater. 46 (1998) 5509.

[8] J. Ning, E.C. Aifantis, Anisotropic and inhomogeneous plastic deformation of polycrystalline solids, in: A.S. Krausz, K. Krausz (Eds.), Unified Constitutive Laws of Plastic Deformation, Academic Press, London, 1996, pp. 319-342.

[9] E.C. Aifantis, J. Eng. Mater. Technol. 106 (1984) 326.

[10] E.C. Aifantis, Int. J. Plast. 3 (1987) 211.

[11] E.C. Aifantis, J. Mech. Behav. Mater. 5 (1994) 335.

[12] N.A. Fleck, G.M. Muller, M.F. Ashby, J.W. Hutchinson, Acta Metall. Mater. 42 (1994) 475.

[13] M.F. Ashby, Philos. Mag. 21 (1970) 399. 